\title{
Sugar-sweetened soft drinks and obesity: a systematic review of the evidence from observational studies and interventions
}

\author{
Sigrid Gibson \\ Sig-Nurture Ltd, 11 Woodway, Guildford, Surrey GU1 2TF, UK
}

\begin{abstract}
Sugar-sweetened soft drinks (SSD) are a special target of many obesity-prevention strategies, yet critical reviews tend to be more cautious regarding the aetiological role of SSD in promoting excess body weight. Since ongoing evaluation of this issue is important, the present systematic review re-examined the evidence from epidemiological studies and interventions, up to July 2008. Database searches of Medline, Cochrane reviews, Google scholar and a hand search of cross-references identified forty-four original studies (twenty-three cross-sectional, seventeen prospective and four intervention) in adults and children, as well as six reviews. These were critically examined for methodology, results and interpretation. Approximately half the crosssectional and prospective studies found a statistically significant association between SSD consumption and BMI, weight, adiposity or weight gain in at least one subgroup. The totality of evidence is dominated by American studies where SSD consumption tends to be higher and formulations different. Most studies suggest that the effect of SSD is small except in susceptible individuals or at high levels of intake. Methodological weaknesses mean that many studies cannot detect whether soft drinks or other aspects of diet and lifestyle have contributed to excess body weight. Progress in reaching a definitive conclusion on the role of SSD in obesity is hampered by the paucity of good-quality interventions which reliably monitor diet and lifestyle and adequately report effect sizes. Of the three long-term ( $>6$ months) interventions, one reported a decrease in obesity prevalence but no change in mean BMI and two found a significant impact only among children already overweight at baseline. Of the six reviews, two concluded that the evidence was strong, one that an association was probable, while three described it as inconclusive, equivocal or near zero. Reasons for some discrepancies are presented.
\end{abstract}

Soft drinks: Sugar: Obesity: Systematic reviews

\section{Introduction}

A number of influential global reports assert that sugarcontaining drinks play a key role in the aetiology of overweight and obesity ${ }^{(1,2)}$. However, comprehensive scientific reviews of the evidence base have tended to be more cautious, highlighting the weaknesses of many studies.

Previous reviews have variously described the evidence as "not conclusivese, '(3), 'equivocal' (4), "probable'(2) and 'strong ${ }^{(5,6)}$. Most recently, a systematic review and metaanalysis concluded that the strength of relationship was near zero and there was evidence of a publication bias towards studies with a positive result ${ }^{(7)}$.

\section{Summary of previous reviews}

The six reviews differ in scope, approach and conclusions. Pereira described the evidence implicating sugar-sweetened drinks in the aetiology of obesity as 'equivocal', criticising the unsatisfactory methodology of many experimental and prospective studies ${ }^{(4)}$. Bachman et al. ${ }^{(3)}$ looked at four proposed mechanisms for the association between sugarsweetened beverages and obesity, including excess energy intake and poorer satiation from liquids. They judged the evidence strongest for the excess energy intake hypothesis but found it "not conclusive ${ }^{\text {(3) }}$. Six of the studies that they included supported the hypothesis but an equal number did not, while those that did had methodological weaknesses such as not controlling for physical activity or using measurements for diet or body weight that had limited reliability and validity.

By contrast, in a systematic review of published studies up to May 2005, Malik et al. concluded that the evidence was 'strong' but conceded that research was needed 'to provide more convergence in the data' and 'to elucidate

Abbreviations: NHANES, National Health and Nutrition Examination Survey; SSD, sugar-sweetened soft drinks.

Corresponding author: Sigrid Gibson, fax + 441483 838018, email sigrid@sig-nurture.com 
mechanisms'(5). Some aspects of this analysis have been criticised, notably the interpretation that the majority of studies show an overall positive association between SSB and obesity ${ }^{(8)}$. Several studies are reported by Malik et al. ${ }^{(5)}$ as positive when only a selected sub-group had a positive result, or classified as 'positive non-significant' where coefficients are near zero and $P$ values in excess of $0 \cdot 2^{(7)}$. Furthermore, the results of two studies were confounded by the inclusion of diet soft drinks.

In 2003, the World Cancer Research Fund ${ }^{(2)}$ began to commission a series of systematic literature reviews (SLR) of food and drinks promoting weight gain. The remit was to assess the totality of evidence; however, it was later decided to exclude both cross-sectional studies and longitudinal observational studies with less than 1 year of follow-up or in children aged under 5 years. The SLR on soft drinks was conducted in 2005 and based on a total of six epidemiological studies including a single randomised controlled trial by James et al. ${ }^{(9)}$. Although the expert group charged with conducting the SLR viewed the evidence as 'limited-suggestive', the panel responsible for interpretation concluded that it was 'probable'. The outcome was a recommendation to 'avoid sugary drinks'(2).

In April 2007, Vartanian et al. ${ }^{(6)}$ published a wideranging systematic review and meta-analysis of the various nutrition and health effects of soft drinks, including fortyfive studies with some measure of body weight or obesity as outcome. For all studies combined, the effect size $(r)$ was 0.08 , which is very small. However, they give more weight to the seven experimental or intervention studies whose effect size was $0 \cdot 24$ (medium).

In contrast to the conclusions reached by Malik et al. ${ }^{(5)}$, Vartanian et al. ${ }^{(6)}$ and the World Cancer Research Fund report $^{(2)}$, the latest meta-analysis, by Forshee et al. ${ }^{(7)}$ concluded that the association between BMI and consumption of sugar-sweetened beverages in children was near zero. The authors also found evidence of a positive publication bias. The review of Forshee et al. ${ }^{(7)}$ included papers published up to October 2006.

Owing to the frequency with which new studies are being published, ongoing review is imperative. This paper presents the results of a comprehensive review of the literature up to July 2008 regarding the association between sugar-containing drinks and body weight and obesity. It does not attempt to cover the literature pertaining to mechanisms or experimental investigations of sugar-sweetened soft drinks' links with energy intake and satiety. In evaluating the strengths and weaknesses of the evidence base, possible reasons for discrepant findings in reviews are highlighted and some issues needing to be addressed in future studies are identified.

\section{Methods}

Papers were identified from web-based searches in PubMed, Google Scholar and the Cochrane Library, supplemented by a hand-search of existing documentation and cross-references. Inclusion criteria were studies and reviews published in English up to July 2008 that related to consumption of sugar-containing drinks (SSD) and their association with body weight, BMI or adiposity in adults or children. The search was conducted iteratively using search terms: 'soft drinks'/'sugar-sweetened beverages'/"soda'/'liquid sugars' with 'weight'/'body weight'/'obesity'/ 'adiposity'. Abstracts were screened for relevance and if they appeared to meet inclusion criteria were imported electronically into a bibliographic database (Endnote version 8; Adept Scientific, Letchworth Garden City, Herts, UK). Full papers were then obtained from the web or requested from authors.

To represent the totality of evidence relating to consumption of soft drinks in a non-laboratory setting, all designs were included (cross-sectional, prospective, and interventions and randomised controlled trial). SSD were defined as all cold beverages containing added sugars, whether carbonated or still, including soda pop (but not diet soda) and fruit squash and drinks with a fruit component less than $100 \%$ pure fruit juice, hot beverages and diet drinks were not included, although studies were included if they assessed these as well as SSD. Studies were excluded if they were animal studies, not published in English, gave no data on consumption of soft drinks or sweetened beverages, provided no anthropometric data, or were short-term experiments or mechanistic studies. Four studies were discarded at this stage because they did not disaggregate SSD data from other sources of sugar ${ }^{(10,11)}$, other snack foods ${ }^{(12)}$ or other interventions ${ }^{(13)}$. Essential details of studies meeting the criteria were extracted into a spreadsheet and classified by design (cross-sectional, longitudinal or intervention study). Study quality was not formally assessed because this was considered too subjective and there is a lack of consensus on criteria for different study designs. Instead the strengths and weaknesses of papers are discussed where these have a direct bearing on the results. Tables $1-5$ summarise these studies and retain the terminology used by the papers (for example, SSD, sweetened beverages, soda, fruit drinks).

\section{Results}

Of a total of forty-four original studies, forty were observational (twenty-three cross-sectional and seventeen prospective) and four were interventions. Three of the prospective studies also provided cross-sectional data at baseline ${ }^{(14-16)}$.

\section{Cross-sectional studies}

Results were equivocal, with less than half of the studies with cross-sectional data ( $n$ 12/27) showing a significant positive association between SSD and BMI or overweight in at least one group (Table 1). Three American prospective studies showed an association at baseline, although in two of these ${ }^{(14,15)}$, results were only significant among females. Several studies using US National Health and Nutrition Examination Survey (NHANES) data were based on the $24 \mathrm{~h}$ recall method for assessing diet, while the large study by Berkey et al. ${ }^{(15)}$ used a semi-quantitative FFQ to assess diet over the previous year. The UK and Irish studies used the 'gold standard' $7 \mathrm{~d}$ weighed dietary record ${ }^{(17,18)}$. 
Table 1. Cross-sectional studies showing a positive association between sugar-sweetened soft drinks (SSD) consumption and BMI or obesity

\begin{tabular}{|c|c|c|c|c|c|c|c|c|}
\hline \multirow[b]{2}{*}{ Study } & \multirow[b]{2}{*}{ Country } & \multirow[b]{2}{*}{$\begin{array}{l}\text { Age group } \\
\text { (years) }\end{array}$} & \multirow[b]{2}{*}{$\begin{array}{l}\text { Subjects } \\
(n)\end{array}$} & \multirow[b]{2}{*}{ Diet method } & \multicolumn{3}{|c|}{ Body weight, BMI or BMI z-score } & \multirow[b]{2}{*}{ Results } \\
\hline & & & & & $\begin{array}{l}\text { Positive } \\
\text { association } \\
(P<0.05)\end{array}$ & Positive but NS & $\begin{array}{l}\text { No association } \\
\text { or negative }\end{array}$ & \\
\hline McCarthy et al. (2006) $)^{(18)}$ & $\begin{array}{l}\text { North and } \\
\text { South Ireland }\end{array}$ & $18-64$ & 1379 & $\begin{array}{l}7 \mathrm{~d} \text { estimated } \\
\text { food diary }\end{array}$ & $\begin{array}{l}\text { 'High energy } \\
\text { beverages' }\end{array}$ & & & $\begin{array}{l}\text { Many foods associated with higher BMI. } \\
\text { Consuming high- } v \text {. low-energy beverages: } \\
\text { OR of obesity } 3.9\end{array}$ \\
\hline Gibson \& Neate $(2007)^{(17)}$ & UK & $4-18$ & 1688 & $\begin{array}{l}7 \mathrm{~d} \text { weighed } \\
\text { record }\end{array}$ & $\begin{array}{l}\text { High SSD } \\
\text { (quintile 5) }\end{array}$ & SSD (overall) & & $\begin{array}{l}\text { Only top quintile SSD (mean } 870 \mathrm{~kJ} / \mathrm{d} \text { ) associated } \\
\text { with overweight (OR } 1.67 ; P=0.03 \mathrm{v} \text {. quintile } \\
\text { 1). Other sources of energy and physical } \\
\text { activity showed stronger associations with BMI }\end{array}$ \\
\hline Liebman et al. (2003) & USA & $18-99$ & 1827 & $24 \mathrm{~h}$ & Soda & & & $\begin{array}{l}\text { Did not distinguish between regular and diet } \\
\text { soda. Contrast }>\text { one soda pop/week } \\
v .<\text { one/week }(P<0.05): 32 \mathrm{v} .20 \% \text { obese in } \\
\text { women; } 27 \mathrm{v} .17 \% \text { in men over } 50 \text { years }\end{array}$ \\
\hline Warner et al. (2006) $)^{(25)}$ & $\begin{array}{l}\text { USA } \\
\text { (Mexican- } \\
\text { Americans) }\end{array}$ & 2 & 385 & $\begin{array}{l}\text { FFQ past 7d } \\
\quad \text { (twenty items) }\end{array}$ & Soda & & & $\begin{array}{l}\text { One or more serving of soda } v \text {. none associated } \\
\text { with odds of obesity of } 3.39(P=0.02) \text { (soda } \\
\text { did not include fruit drinks) }\end{array}$ \\
\hline $\begin{array}{l}\text { French et al. (1994) } \\
\quad \text { (prospective study with } \\
\text { additional cross-sectional } \\
\text { analysis) }\end{array}$ & USA & Adults & 3552 & $\mathrm{FFQ}$ & $\begin{array}{l}\text { Soda } \\
\quad \text { (women) }\end{array}$ & Soda (men) & & $\begin{array}{l}\text { Women consuming one serving/week were } \\
0.47 \mathrm{lbs} \text { heavier than non-consumers } \\
(P=0.03) \text {. Men were } 0.33 \mathrm{lbs} \text { heavier } \\
(P=0.13)\end{array}$ \\
\hline Novotny et al. (2004) $)^{(23)}$ & Hawaii & $9-14$ & 323 & $\begin{array}{l}3 \mathrm{~d} \text { semi-weighed } \\
\text { record }\end{array}$ & Soda & & & $\begin{array}{l}\text { Body weight difference equivalent to } 1.7 \mathrm{~kg} \text { per } \\
350 \mathrm{ml} \text { can of soda) in multiple regression } \\
(P=0.01) \text {. Did not include fruit drinks }\end{array}$ \\
\hline Ariza et al. (2004) & USA (Hispanic) & $5-6$ & 80 & $\begin{array}{l}\text { Interview questioned } \\
\text { frequency of SSD }\end{array}$ & SSD & & & $\begin{array}{l}\text { Overweight children were more likely to consume } \\
\text { sweetened beverages (powdered drinks, soda } \\
\text { pop) daily ( } 67 \mathrm{v} .39 \% ; P=0.03 \text { ) }\end{array}$ \\
\hline Gillis \& Bar-Or (2003) $)^{(22)}$ & Canada & $4-16$ & 181 & $24 h+F F Q$ & SSD & & & $\begin{array}{l}\text { Obese consumed more soda/SSD (seven } v \text {. five } \\
\text { servings/week; } P<0.05 \text { for both sexes } \\
\text { combined but NS in girls) }\end{array}$ \\
\hline $\begin{array}{l}\text { Ludwig et al. (2001) } \\
\quad \text { (prospective study with } \\
\text { additional cross-sectional } \\
\text { analysis) }\end{array}$ & USA & 12 & 548 & $\begin{array}{l}\text { Youth FFQ } \\
\text { (past month) }\end{array}$ & SSD & & & $\begin{array}{l}\text { Baseline SSD servings positively associated with } \\
\text { change in BMI (mean } 0.18 \mathrm{~kg} / \mathrm{m}^{2} \text { per serving; } \\
P=0.02 \text { ) }\end{array}$ \\
\hline $\begin{array}{l}\text { Berkey et al. (2004) } \\
\quad \text { (prospective study with } \\
\text { additional cross-sectional } \\
\text { analysis) }\end{array}$ & USA & $9-14$ & 16679 & $\begin{array}{l}\text { FFQ semi-quantitative. } \\
\text { Typical intake over } \\
\text { past year and change }\end{array}$ & SSD (girls) & & & $\begin{array}{l}\text { Baseline BMI }+0.06 \mathrm{~kg} / \mathrm{m}^{2} \text { per serving of SSD in } \\
\text { girls }(P=0.04) . S S D \text { associated with higher } \\
\text { total energy intakes }(216 \mathrm{kcal} / \mathrm{serving})\end{array}$ \\
\hline Troiano et al. $(2000)^{(19)}$ & USA & $2-19$ & 10371 & $24 \mathrm{~h}$ recall & SSD energy & & & $\begin{array}{l}\text { Except for females aged } 12-19 \text { years, overweight } \\
\text { children in all age groups had soft drink intakes } \\
\text { about } 2 \% \text { higher (as a percentage of energy) } \\
\text { than normal-weight children }\end{array}$ \\
\hline Nicklas et al. $(2003)^{(20)}$ & USA & 10 & 1562 & $24 \mathrm{~h}$ recall & $\begin{array}{l}\text { Sweetened } \\
\quad \text { beverages } \\
(P<0.001)\end{array}$ & & & $\begin{array}{l}\text { Consumption of sweetened beverages }(58 \% \text { soft } \\
\text { drinks, } 20 \% \text { fruit-flavour drinks, } 19 \% \text { tea and } \\
3 \% \text { coffee) was positively associated with } \\
\text { overweight status }(P<0.001) \text { (OR 1.33) }\end{array}$ \\
\hline
\end{tabular}


WS Nutrition Research Reviews

Table 2. Cross-sectional studies showing a non-significant or null association between sugar-sweetened soft drinks (SSD) consumption and BMI or obesity

\begin{tabular}{|c|c|c|c|c|c|c|c|c|}
\hline \multirow[b]{2}{*}{$\begin{array}{l}\text { Study } \\
\text { Andersen } \\
\quad \text { et al. (2005) }\end{array}$} & \multirow[b]{2}{*}{$\begin{array}{l}\text { Country } \\
\text { Norway }\end{array}$} & \multirow[b]{2}{*}{$\begin{array}{l}\text { Age group } \\
\text { (years) } \\
8-14\end{array}$} & \multirow[b]{2}{*}{$\begin{array}{l}\text { Subjects } \\
(n) \\
3139\end{array}$} & \multirow[b]{2}{*}{$\begin{array}{l}\text { Diet method } \\
4 \mathrm{~d} \text { food diary }\end{array}$} & \multicolumn{3}{|c|}{ Body weight, BMI or BMI z-score } & \multirow[b]{2}{*}{$\begin{array}{c}\text { Results } \\
\text { No association between overweight status and i } \\
\text { ntake of sweetened soft drinks or \% energy } \\
\text { from sugars. Inverse association with sweets }\end{array}$} \\
\hline & & & & & $\begin{array}{l}\text { Positive } \\
\text { association } \\
(P<0.05)\end{array}$ & $\begin{array}{l}\text { Positive } \\
\text { but NS }\end{array}$ & $\begin{array}{l}\text { No association } \\
\text { or negative } \\
\text { SSD }\end{array}$ & \\
\hline $\begin{array}{l}\text { Bandini } \\
\text { et al. }(1999)^{(34)}\end{array}$ & USA & $12-18$ & 43 & & & & Soda & $\begin{array}{l}\text { No difference in percentage of energy from soda } \\
\text { between obese }(5.9 \%) \text { and non-obese }(6.0 \%)\end{array}$ \\
\hline $\begin{array}{l}\text { Forshee \& } \\
\quad \text { Storey }(2003)^{(30)}\end{array}$ & USA & $6-19$ & 3311 & CSFII $(2 \times 24 h)$ & & $\begin{array}{l}\text { Diet soda } \\
\text { (girls) }\end{array}$ & Soda & $\begin{array}{l}\text { No association with regular soda }(P>0.2) \text {; slight } \\
\text { positive association with diet soda in girls }\end{array}$ \\
\hline $\begin{array}{l}\text { Forshee } \\
\quad \text { et al. }(2004)^{(31)}\end{array}$ & USA & $12-16$ & 2216 & $\begin{array}{l}24 \mathrm{~h}+\mathrm{FFQ} \\
\quad \text { (NHANES III) }\end{array}$ & & & $\begin{array}{l}\text { Soda and } \\
\text { fruit ades }\end{array}$ & $\begin{array}{l}\text { SSD (soda) serving of } 370 \mathrm{~g} \text { associated with } \\
+0.26 \mathrm{~kg} / \mathrm{m}^{2} \mathrm{BMI} \text { in girls and } 0.11 \mathrm{~kg} / \mathrm{m}^{2} \text { in } \\
\text { boys; } \mathrm{NS}(P>0.3) \text {. }\end{array}$ \\
\hline $\begin{array}{l}\text { Giammattei } \\
\quad \text { et al. }(2003)^{(27)}\end{array}$ & USA & $11-13$ & 385 & $\begin{array}{l}\text { Self-administered } \\
\text { lifestyle } \\
\text { and habits } \\
\text { questionnaire }\end{array}$ & Diet soda & SSD & & $\begin{array}{l}\text { NS positive correlation for regular soda }(r 0.10 \\
\begin{array}{l}P=0.08) \text {. Positive association with diet soda } \\
(r 0.19 ; P=0.01)\end{array}\end{array}$ \\
\hline Gibson $(1998)^{(36)}$ & UK & $1-4$ & 1546 & $4 \mathrm{~d}$ weighed record & & & SSD & $\begin{array}{l}\text { No association between } \mathrm{BMI} \text { and amount or } \\
\text { proportion of energy from soft drinks }(P>0.50)\end{array}$ \\
\hline $\begin{array}{l}\text { Janssen } \\
\quad \text { et al. }(2005)^{(28)}\end{array}$ & $\begin{array}{l}\text { Thirty-four } \\
\text { countries }\end{array}$ & $10-16$ & 137000 & FFQ & & & SSD & $\begin{array}{l}\text { Association with SSD NS in thirty countries, positive } \\
\text { in two, negative in two }\end{array}$ \\
\hline $\begin{array}{l}\text { O'Connor } \\
\text { et al. }(2006)^{(32)}\end{array}$ & USA & $2-5$ & 1160 & $24 \mathrm{~h}$ & & & SSD & $\begin{array}{l}\text { No association with the amount of total beverages, } \\
\text { milk, } 100 \% \text { fruit juice, fruit drink, or soda } \\
\text { consumed }\end{array}$ \\
\hline $\begin{array}{l}\text { Rajeshwari } \\
\quad \text { et al. }(2005)^{(21)}\end{array}$ & USA & 10 & 1548 & $24 \mathrm{~h}$ & & & SSD & $\begin{array}{l}\text { No linear relationship between sweetened-beverage } \\
\text { consumption and BMI. BMI increased from } 1970 \mathrm{~s} \\
\text { to } 1990 \mathrm{~s} \text { across all SSD consumption groups. } \\
\text { Energy did not }\end{array}$ \\
\hline $\begin{array}{l}\text { Rodriguez-Artalejo } \\
\text { et al. }(2003)^{(37)}\end{array}$ & Spain & $6-7$ & 1112 & $\mathrm{FFQ}$ & & & SSD & No association between SSD and BMI \\
\hline $\begin{array}{l}\text { Roseman } \\
\quad \text { et al. }(2007)^{(33)}\end{array}$ & USA & $11-14$ & 4049 & $7 \mathrm{~d}$ recall & & & Soft drinks & $\begin{array}{l}\text { No significant association between soft drink } \\
\text { consumption and students' weight status. } \\
\text { Definition not given. No } P \text { values }\end{array}$ \\
\hline $\begin{array}{l}\text { Silveira } \\
\quad \text { et al. }(2006)^{(38)}\end{array}$ & Brazil & $14-19$ & 172 & $\begin{array}{l}4 \mathrm{~d} \text { diary, FFQ, } \\
\text { lifestyle } \\
\text { questionnaire }\end{array}$ & & & SSD & $\begin{array}{l}\text { Daily soft drink consumption was not associated with } \\
\text { overweight (OR } 0.69 \text { ( } 95 \% \mathrm{Cl} 0.27,1.72) \\
\quad P=0.377 ; 13 \% \text { of cases } v .18 \% \text { of controls) }\end{array}$ \\
\hline $\begin{array}{l}\text { Sun \& Empie } \\
\quad(2007)^{(40)}\end{array}$ & USA & $20-74$ & 38000 & $\begin{array}{l}\text { Combined CSFII } \\
\text { and NHANES }\end{array}$ & & & SSD & $\begin{array}{l}\text { No substantive differences in BMI and obesity } \\
\text { occurrence between frequent and infrequent } \\
\text { users of SSD. Only in NHANES 1999-2002, } \\
\text { obese adults consumed } 72 \mathrm{~g} / \mathrm{d} \text { more (31 kcal) } \\
\text { than the mean for non-obese adults }\end{array}$ \\
\hline $\begin{array}{l}\text { Veugelers \& } \\
\quad \text { Fitzgerald } \\
(2005)^{(39)}\end{array}$ & Canada & $10-11$ & 4298 & $\begin{array}{l}\text { FFQ; soft drink } \\
\text { sales in school }\end{array}$ & & & SSD & $\begin{array}{l}\text { Availability of soft drinks at schools was not } \\
\text { associated with risk of overweight (OR } 0.99) \text {, } \\
\text { although consumption was higher in schools } \\
\text { that sold soft drinks ( } 4.0 \text { v. } 3.6 \text { cans per week; } \\
P=0.01 \text { ) }\end{array}$ \\
\hline
\end{tabular}




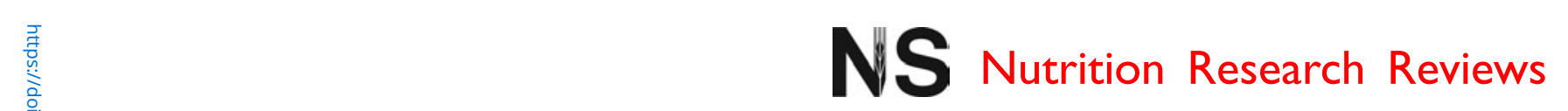

Table 3. Longitudinal studies showing a positive association between sugar-sweetened soft drinks (SSD) consumption and BMI or obesity

\begin{tabular}{|c|c|c|c|c|c|c|c|c|c|}
\hline \multirow[b]{2}{*}{ Study } & \multirow[b]{2}{*}{ Country } & \multirow[b]{2}{*}{$\begin{array}{l}\text { Age group } \\
\text { (years) }\end{array}$} & \multirow[b]{2}{*}{$\begin{array}{l}\text { Subjects } \\
\qquad(n)\end{array}$} & \multirow[b]{2}{*}{ Follow-up } & \multirow[b]{2}{*}{ Diet method } & \multicolumn{3}{|c|}{ Body weight } & \multirow[b]{2}{*}{ Results } \\
\hline & & & & & & $\begin{array}{l}\text { Positive } \\
\text { association } \\
(P<0.05)\end{array}$ & $\begin{array}{l}\text { Positive } \\
\text { but NS }\end{array}$ & $\begin{array}{l}\text { No association } \\
\text { or negative }\end{array}$ & \\
\hline $\begin{array}{l}\text { Nooyens et al. } \\
\quad(2005)^{(43)}\end{array}$ & Netherlands & $\begin{array}{r}50-65 \\
\text { Men }\end{array}$ & 288 & 5 years & $\begin{array}{l}\mathrm{FFQ} \\
\quad(\mathrm{EPIC})\end{array}$ & SSD & & & $\begin{array}{l}\text { Unadjusted model: change in } \mathrm{BMI}=0.2 \mathrm{~kg} / \mathrm{m}^{2} \text { per year } \\
\text { per serving per d; waist } 0.16 \mathrm{~cm} / \text { year per serving } \\
\text { per d (in adjusted model BMI change }=0.12 \mathrm{~kg} / \text { year } \\
\text { per serving per d; waist } 0.06 \mathrm{~cm} / \text { year) }\end{array}$ \\
\hline $\begin{array}{l}\text { Welsh et al. } \\
\qquad(2005)^{(42)}\end{array}$ & USA & 2 and 3 & 10904 & 1 year & $\mathrm{FFQ}$ & $\begin{array}{l}\text { SSD } \\
\quad \text { (overweight) }\end{array}$ & $\begin{array}{l}\text { SSD (normal } \\
\text { weight) }\end{array}$ & & $\begin{array}{l}\text { Positive association between SSD and overweight only } \\
\text { in children overweight }(>85 \text { th percentile BMI) at } \\
\text { baseline. OR about } 2 \text { for }>1 \text { drink/d } v .<1 \text { drink/d } \\
\text { (referent) }\end{array}$ \\
\hline $\begin{array}{l}\text { Berkey et al. } \\
\qquad(2004)^{(15)}\end{array}$ & USA & $9-14$ & $>12000$ & 2 years & $\mathrm{FFQ}$ & SSD (boys) & SSD (girls) & & $\begin{array}{l}\text { Consumption of sugar-added beverages was associated } \\
\text { with small } \mathrm{BMl} \text { gains during the corresponding year } \\
\text { (boys: }+0.03 \mathrm{~kg} / \mathrm{m}^{2} \text { per daily serving, } P=0.04 ; \text { girls: } \\
\left.+0.02 \mathrm{~kg} / \mathrm{m}^{2}, P=0.096\right) \text {. Boys who increased } \\
\text { consumption of sugar-added beverages from the } \\
\text { prior year experienced weight gain }\left(+0.04 \mathrm{~kg} / \mathrm{m}^{2}\right. \\
\text { per additional daily serving; } P=0.01)\end{array}$ \\
\hline $\begin{array}{l}\text { Bes-Rastrollo } \\
\text { et al. } \\
(2006)^{(41)}\end{array}$ & Spain & Adults & 7194 & 2 years & $\mathrm{FFQ}$ & $\begin{array}{l}\text { SSD (in previous } \\
\text { weight-gainers) }\end{array}$ & & $\begin{array}{l}\text { SSD (in } \\
\text { weight- } \\
\text { stable) }\end{array}$ & $\begin{array}{l}\text { Among participants who had gained }>3 \mathrm{~kg} \text { in the } 5 \text { years } \\
\text { before baseline, adjusted odds of subsequent } \\
\text { weight gain in quintile } 5 \mathrm{v} \text {. quintile } 1 \text { of } \\
\text { sugar-sweetened soft drink consumption was } 1.6 \\
(P=0.02)\end{array}$ \\
\hline $\begin{array}{l}\text { Ludwig et al. } \\
\qquad(2001)^{(16)}\end{array}$ & USA & 12 & 548 & 19 months & $\begin{array}{l}\text { Youth FFQ } \\
\text { (past } \\
\text { month) }\end{array}$ & SSD & & & $\begin{array}{l}\text { BMI increased } 0.24 \mathrm{~kg} / \mathrm{m}^{2} \text { per serving }(P=0.03) \\
\text { (adjusted for baseline BMl, demographic, diet and } \\
\text { lifestyle). Frequency of obesity based on thirty-seven } \\
\text { cases of incident obesity (OR } 1.60(95 \% \mathrm{Cl} 1.14 \\
2.24) ; P=0.02)\end{array}$ \\
\hline $\begin{array}{l}\text { Phillips et al. } \\
\qquad(2004)^{(46)}\end{array}$ & USA & $\begin{array}{l}\text { 8-12, } \\
\text { Girls }\end{array}$ & $\begin{array}{l}196 \text { enrolled } \\
\text { (178 after } \\
\text { exclusion } \\
\text { of } 18)\end{array}$ & 7 years & $\begin{array}{l}\text { FFQ (past } \\
\text { year) }\end{array}$ & SSD & & & $\begin{array}{l}\text { Percentage energy from soda associated with higher } \\
\text { BMI } z \text {-score (quartile } 3(>1.5 \%) \text { and quartile } 4 \\
(>3.2 \%) \text { had BMl } z \text {-score }+0.17 \text { compared with } \\
\text { quartile 1) }\end{array}$ \\
\hline $\begin{array}{l}\text { Schulze et al. } \\
\qquad(2004)^{(47)}\end{array}$ & USA & $\begin{array}{l}\text { Adult } \\
\quad \text { women }\end{array}$ & 51000 & 8 years & - & SSD & & & $\begin{array}{l}\text { Weight gain in } 1007 \text { women increasing SSD } \\
\text { consumption from }<1 / \text { week to }>1 / \mathrm{d} \text { was about } 3 \mathrm{~kg} \\
\text { (over } 4 \text { years) more than in those } 1020 \text { women who } \\
\text { decreased SSD by the same amount from } 1995 \text { to } \\
1999 \text {. Weight gain also about } 3 \mathrm{~kg} \text { in those who } \\
\text { were consistent high or low consumers }\end{array}$ \\
\hline $\begin{array}{l}\text { Striegel-Moore } \\
\quad \text { et al. } \\
(2006)^{(44)}\end{array}$ & USA & $\begin{array}{l}9-10, \\
\text { Girls }\end{array}$ & 3371 & 9 years & $\begin{array}{l}3 \mathrm{~d} \text { records } \\
\text { annually }\end{array}$ & SSD & & & $\begin{array}{l}\text { Of all beverages, increasing soda consumption } \\
\text { predicted the greatest increase of BMI }\end{array}$ \\
\hline
\end{tabular}

EPIC, European Prospective Investigation into Cancer and Nutrition. 


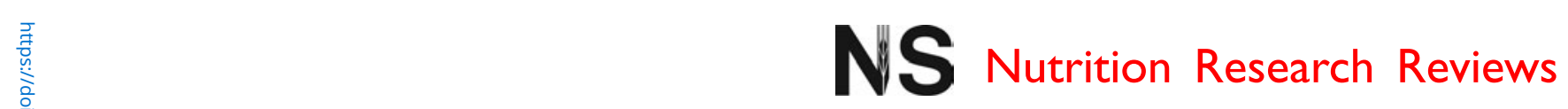

Table 4. Longitudinal studies showing a non-significant or null association between sugar-sweetened soft drinks (SSD) consumption and BMI or obesity

\begin{tabular}{|c|c|c|c|c|c|c|c|c|c|}
\hline \multirow[b]{2}{*}{ Study } & \multirow[b]{2}{*}{ Country } & \multirow[b]{2}{*}{$\begin{array}{l}\text { Age group } \\
\text { (years) }\end{array}$} & \multirow[b]{2}{*}{$\begin{array}{l}\text { Subjects } \\
(n)\end{array}$} & \multirow[b]{2}{*}{ Follow-up } & \multirow[b]{2}{*}{ Diet method } & \multicolumn{3}{|c|}{ Body weight } & \multirow[b]{2}{*}{ Results } \\
\hline & & & & & & $\begin{array}{c}\text { Positive } \\
\text { association } \\
(P<0.05)\end{array}$ & $\begin{array}{l}\text { Positive } \\
\text { but NS }\end{array}$ & $\begin{array}{c}\text { No } \\
\text { association } \\
\text { or negative }\end{array}$ & \\
\hline $\begin{array}{l}\text { Libuda et al. } \\
\qquad(2007)^{(48)}\end{array}$ & Germany & $9-18$ & 244 & 5 years & $3 \mathrm{~d}$ WDR & & SSD (girls) & SSD (boys) & $\begin{array}{l}\text { SSD had weaker association than } \\
\text { fruit juice. Change in total } \\
\text { 'energetic beverages' was } \\
\text { significantly associated with } \\
\text { change in BMI but not } \\
\text { percentage body fat. } \\
\text { Girls: change }=0.055 \mathrm{BMI} \\
\text { standard deviations/MJ } \\
\text { SSD }(P=0.08)\end{array}$ \\
\hline $\begin{array}{l}\text { Johnson et al. } \\
(2007)^{(52)}\end{array}$ & UK & $5-7,9$ & 1203 & 2 years & $\begin{array}{c}3 d \text { NWDR at } \\
\text { age } 5 \text { and } \\
7 \text { years }\end{array}$ & $\begin{array}{l}\text { Diet soft } \\
\quad \text { drinks }^{*}\end{array}$ & & $\mathrm{SSD}^{*}$ & $\begin{array}{l}\text { No association between SSD intake } \\
\text { at age } 5 \text { or } 7 \text { years and BMI at } \\
\text { age } 9 \text { years. Inverse association } \\
\text { between SSD and fat mass. } \\
\text { Small positive association with } \\
\text { low-energy drinks }\end{array}$ \\
\hline $\begin{array}{l}\text { Blum et al. } \\
(2005)^{(53)}\end{array}$ & USA & $\begin{array}{l}\text { Elementary } \\
\text { school } \\
\text { children }\end{array}$ & 166 & 2 years & $24 \mathrm{~h}$ recall & & & SSD & $\begin{array}{l}\text { No association between SSD and } \\
\text { year } 2 \text { BMI } z \text {-score }(\beta=-0.003, \\
\text { se } 0.004 ; P>0.05) \text {. Only diet } \\
\text { drinks positively associated } \\
\text { with BMI }\end{array}$ \\
\hline $\begin{array}{l}\text { Kvaavik et al. } \\
\quad(2005)^{(55)}\end{array}$ & Norway & 15,33 & 485 & 8 years & Questionnaires & & & SSD & $\begin{array}{l}\text { No differences in BMI, overweight } \\
\text { or obesity in } 1999 \text { between } \\
\text { long-term high and low } \\
\text { consumers of SSD }\end{array}$ \\
\hline $\begin{array}{l}\text { Mundt et al. } \\
(2006)^{(51)}\end{array}$ & Canada & $8-19$ & 208 & 5 years & $\begin{array}{l}24 \mathrm{~h} \text { recall } \\
\text { repeated }\end{array}$ & & & $\mathrm{SSD}^{*}$ & $\begin{array}{l}\text { SSD was not related to fat mass } \\
\text { development or physical activity }\end{array}$ \\
\hline $\begin{array}{l}\text { Newby et al. } \\
(2004)^{(54)}\end{array}$ & USA & $2-5$ & 1345 & $\begin{array}{l}6-12 \\
\text { months }\end{array}$ & & & & SSD & $\begin{array}{l}\text { No association between any type } \\
\text { of beverage (soda, diet soda, } \\
\text { juice, milk) and change in BMI } \\
\text { in multivariate adjusted models }\end{array}$ \\
\hline $\begin{array}{l}\text { Schulz et al. } \\
(2002)^{(50)}\end{array}$ & $\begin{array}{l}\text { Germany } \\
\text { (EPIC) }\end{array}$ & Adults & 17369 & 2 years & FFQ & & & $\begin{array}{l}\text { Soft drinks } \\
\quad \text { combined }\end{array}$ & $\begin{array}{l}\text { Soft drinks included water and juice. } \\
\text { Mixed results for men (higher } \\
\text { 'soft drink' intake at baseline was } \\
\text { very weakly associated with both } \\
\text { losses and gains) and weakly } \\
\text { negative association for women } \\
\text { (slightly greater risk of large } \\
\text { weight losses; OR } 1.02 \text { (95\% } \\
\text { Cl 1.00, 1.03)) }\end{array}$ \\
\hline $\begin{array}{l}\text { French et al. } \\
\qquad(1994)^{(14)}\end{array}$ & USA & Adults & 3552 & 2 years & FFQ & & $\begin{array}{l}\text { Soda } \\
\qquad(P>0.2)\end{array}$ & & $\begin{array}{l}\text { Small NS association between } \\
\text { soda intake and change in } \\
\text { weight over } 2 \text { years per serving } \\
(12 \mathrm{oz}) \text { (women } 0.08 \mathrm{lbs} \\
(P=0.39) ; \text { men } 0.11 \mathrm{lbs} \\
(P=0.22))\end{array}$ \\
\hline $\begin{array}{l}\text { Mrdjenovic \& } \\
\text { Levitsky } \\
(2003)^{(49)}\end{array}$ & & $6-13$ & $\begin{array}{l}30 \text { (final } \\
\text { sample 21) }\end{array}$ & $4-8$ weeks & & & $\stackrel{S S D}{(>16 o z)}$ & & $\begin{array}{l}\text { No significant difference in weight } \\
\text { gain in high SSD }(>16 \mathrm{oz} / \mathrm{d}) \\
\text { v. lower SSD }(6-16 \mathrm{oz}) \\
(1.12 \mathrm{v} .0 .32-0.48 \mathrm{~kg} ; P=0.4)\end{array}$ \\
\hline
\end{tabular}


Table 5. Intervention studies of sugar-sweetened soft drinks (SSD) consumption and BMI or obesity

\begin{tabular}{|c|c|c|c|c|c|c|c|c|c|}
\hline \multirow[b]{2}{*}{ Study } & \multirow[b]{2}{*}{ Country } & \multirow[b]{2}{*}{$\begin{array}{c}\text { Age } \\
\text { (years) }\end{array}$} & \multirow[b]{2}{*}{ Subjects $(n)$} & \multirow[b]{2}{*}{$\begin{array}{l}\text { Intervention } \\
\text { and duration }\end{array}$} & \multirow[b]{2}{*}{ Diet method } & \multicolumn{3}{|c|}{ Body weight } & \multirow[b]{2}{*}{ Results } \\
\hline & & & & & & $\begin{array}{c}\text { Positive } \\
\text { association } \\
(P<0.05)\end{array}$ & $\begin{array}{l}\text { Positive } \\
\text { but NS }\end{array}$ & $\begin{array}{c}\text { NS or } \\
\text { negative }\end{array}$ & \\
\hline $\begin{array}{l}\text { Sichieri et al. } \\
\quad(2008)^{(57)}\end{array}$ & Brazil & $9-12$ & 1140 & $\begin{array}{l}\text { School-based } \\
\text { intervention to } \\
\text { reduce consumption } \\
\text { of SSD over } 1 \text { year }\end{array}$ & $\begin{array}{l}24 \mathrm{~h} \text { recall at } \\
\text { baseline } \\
\text { and follow-up }\end{array}$ & $\begin{array}{l}\text { Overweight girls } \\
\text { ( } n \text { about }<80 \text { ) } \\
\text { (unadjusted } \\
\text { model: } \\
\text { BMI }-0.01 \text {; } \\
P=0.009 \text { ) }\end{array}$ & $\begin{array}{l}\text { Boys and girls } \\
\text { overweight at } \\
\text { baseline } \\
\text { (adjusted } \\
\text { for age: } \\
\text { BMI }-0.007 \\
P=0.11 \text { ) }\end{array}$ & Total sample & $\begin{array}{l}\text { SSD consumption reduced by } \\
69 \mathrm{ml} / \mathrm{d} \text { in intervention } v \text {. } \\
13 \mathrm{ml} \text { in controls (difference } \\
56 \mathrm{ml} ; P=0.03 \text { ). } \\
\text { No significant difference in } \\
\text { BMI (both groups gained). } \\
\text { Unadjusted BMl in girls } \\
\text { overweight at baseline }(15 \%) \\
-0.4 \mathrm{~kg} / \mathrm{m}^{2} \text { in intervention } \\
v .-0.2 \mathrm{~kg} / \mathrm{m}^{2} \text { in controls } \\
(P=0.009) \text {. } \\
\text { Adjusted model: BMl }-0.007 \\
(P=0.11)\end{array}$ \\
\hline $\begin{array}{l}\text { Ebbeling et al. } \\
\qquad(2006)^{(56)}\end{array}$ & USA & $13-18$ & 113 & $\begin{array}{l}\text { Home delivery of } \\
\text { diet soft drinks } \\
\text { to replace normal } \\
\text { consumption } \\
\text { for } 6 \text { months }\end{array}$ & $\begin{array}{l}2 \times 24 \mathrm{~h} \text { diet recall } \\
\text { and activity } \\
\text { diary at baseline } \\
\text { and follow-up }\end{array}$ & $\begin{array}{l}\text { SSB (high } \\
\quad \text { baseline BMI) }\end{array}$ & & SSB (overall) & $\begin{array}{l}82 \% \text { reduction in SSB in } \\
\text { intervention group, no change } \\
\text { in controls ( } 250 \mathrm{kcal} \text { energy } \\
\text { difference). BMI decreased } \\
\text { on average by } 0.26 \mathrm{~kg} / \mathrm{m}^{2} \text { for } \\
\text { every serving per d of SSB } \\
\text { that was displaced. } \\
\text { For top tertile BMl at baseline, } \\
\text { BMl change }=-0.75 \mathrm{~kg} / \mathrm{m}^{2} \\
(P=0.03) \text {. Change in total } \\
\text { group BMI }-0.14 \text { units (NS) }\end{array}$ \\
\hline $\begin{array}{l}\text { James et al. } \\
(2004)^{(9)}\end{array}$ & UK & $7-11$ & 644 & $\begin{array}{l}\text { School-based lessons } \\
\text { (Ditch the Fizz) } \\
\text { given five times } \\
\text { over } 1 \text { year }\end{array}$ & $\begin{array}{l}\text { Beverage diaries } \\
\text { (non-validated) }\end{array}$ & Soda (all types) & & & $\begin{array}{l}\text { Soda consumption lowered in } \\
\text { intervention group by } 0.7 \\
\text { servings over } 3 \text { d. Prevalence } \\
\text { of obesity was } 7.7 \% \text { lower in } \\
\text { intervention group but no } \\
\text { difference in mean BMI }\end{array}$ \\
\hline $\begin{array}{l}\text { Raben et al. } \\
(2002)^{(60)}\end{array}$ & Denmark & Adults & $\begin{array}{l}41 \text { (21 } \\
\text { intervention, } \\
20 \text { controls) }\end{array}$ & $\begin{array}{l}10 \text { weeks of } \\
\text { high-sucrose diet } \\
\text { (>60\% from SSD) } \\
\text { or aspartame- } \\
\text { containing drinks }\end{array}$ & $\begin{array}{l}7 \mathrm{~d} \text { records and } 7 \mathrm{~d} \\
\text { diaries at } 0,5 \text { and } \\
10 \text { weeks, eating } \\
\text { behaviour } \\
\text { questionnaire } \\
\text { at week } 10\end{array}$ & $\begin{array}{l}\text { High-sugar diet } \\
\text { (28\% sucrose, } \\
\text { of which drinks } \\
\text { provided } \\
\text { about } 60 \%)\end{array}$ & & & $\begin{array}{l}\text { High-sugar diet ( } 28 \% \text { energy } \\
\text { from sucrose) provided an } \\
\text { extra } 3.4 \mathrm{MJ} \text { energy but there } \\
\text { was some compensation } \\
\text { (1.6 MJ difference in total } \\
\text { energy). Body-weight gain in } \\
\text { sucrose group }=1.6 \mathrm{~kg}, \\
\text { controls lost } 1 \mathrm{~kg} \text {. Weight } \\
\text { gain in intervention group } \\
\text { was half the predicted amount } \\
\text { based on energy intake }\end{array}$ \\
\hline
\end{tabular}


Studies showing a positive association with BMI in children. The majority of studies on children and youth derive from the USA, where consumption of SSD is typically about twice that of UK and Europe $(8-10 \%$ of energy $v .4-5 \%$ ). The largest of these was the prospective study by Berkey et al. of 16679 children, which found a significant association between baseline SSD consumption and BMI in girls and a non-significant association among boys ${ }^{(15)}$. The prospective study of Ludwig et al. also found an association between baseline consumption of sugary drinks and change in BMI over 19 months (mean 0.18 units for each daily serving (95\% CI 0.09, 0.27); $P=0.02)^{(16)}$. Troiano et al. ${ }^{(19)}$ using USA NHANES data spanning the 20 years up to 1994 reported a higher consumption of energy from soft drinks among overweight youth (2-19 years) compared with non-overweight youth in each age group. However, lack of evidence of an increase in energy intake suggested that physical inactivity was a major factor ${ }^{(19)}$. In the Bogalusa study of 10-year-olds in Louisiana ( $n$ 1594) Nicklas et al. reported that consumption of sweetened beverages (a definition which included tea and coffee) was associated with risk of overweight, but so were numerous other eating patterns ${ }^{(20)}$. They noted that the percentage of variance explained by any of the eating patterns was very small and results varied by ethnic group and sex. A subsequent trend analysis of this same dataset concluded that there was no linear association between BMI and SSD because BMI increased from the 1970 s to the 1990 s across all SSD consumption groups ${ }^{(21)}$. In a small case-control study of 181 Canadian children (ninety obese and ninetyone matched controls), obese children reported a higher frequency of consuming regular soda (seven $v$. five times per week; $P<0.05$ ) but also consumed more energy, fat, meat, chips and grains as well as more food away from home ${ }^{(22)}$. However, one criticism of this study is that the non-obese controls were self-selected volunteers who may have been from more health-conscious families.

Positive associations of SSD with weight status may be more likely in populations with high intakes, including some ethnic groups. A Hawaiian study among 9- to 14-yearolds ( $n$ 323) who were high consumers of soda $(750 \mathrm{~g} / \mathrm{d})$ found each serving to be associated with an additional $1.7 \mathrm{~kg}$ body weight $(P=0.01)$ while milk was inversely associated with weight ${ }^{(23)}$. This study used a $3 \mathrm{~d}$ record of diet and adjusted for all main confounders such as puberty, physical activity, energy intake and ethnicity. Evidence from two other studies is weaker. In a pilot study among Hispanic 5- to 6 -year-olds ${ }^{(24)}$ the overweight children were more likely to consume sweetened beverages daily (67 v. 39\%; $P=0.03$ ) but only eighty out of 250 mothers completed an interview and few other components of diet were assessed. Among Mexican-American infants whose mothers were recruited in pregnancy, the adjusted odds of being overweight at 2 years were three times higher for those who consumed soda daily compared with none ${ }^{(25)}$. One weakness of this study is that fruit drinks, which are popular in this young age group, were not assessed.

Last, an analysis of UK data from a national survey of young people aged 4-18 years found a weak association between the risk of overweight and SSD consumption that only reached statistical significance for the top quintile (mean $870 \mathrm{~kJ} / \mathrm{d}$ from SSD; OR 1.67 (95\% CI 1.04, 2.66); $P=0.03)^{(17)}$. However, other sources of energy intake and physical activity had a stronger impact in regression models. Importantly, this study also incorporated adjustments for under-reporting and dieting.

Studies showing a positive association with BMI in adults. There were three studies in adult populations showing a significant positive association between SSD and obesity. Like some studies in children, many of these suggest that the association is more complex, with clustering of lifestyle and environmental factors in certain groups. In the largest study, a prospective investigation of 3552 American adults in the Healthy Worker Project, women consuming one serving per week of soda were $0.21 \mathrm{~kg}$ heavier at baseline than non-consumers $(P=0.03)$, but men consuming one serving $v$. none were not significantly heavier $(0.15 \mathrm{~kg} ; P=0 \cdot 13)^{(14)}$. In a study of rural communities in the USA, Liebman et al. ${ }^{(26)}$ found that subjects who drank one or more servings of soda pop per week were more likely to be overweight or obese than those who drank less, while other sweetened beverages such as Kool Aid and fruit juices showed no relation with overweight. However, they also found that overweight or obese adults were more likely to order supersize portions, watch TV and to eat while doing other activities ${ }^{(26)}$. Second, as this study did not distinguish between regular and diet versions of soda, there may be some overestimation due to reverse causality (diet drinks being chosen by overweight people). Among Irish adults, McCarthy et al. found that consuming high-energy beverages rather than low-energy beverages was associated with a higher likelihood of being obese but so was consuming larger portions of many foods, such as chips, savouries and fat spreads ${ }^{(18)}$. Importantly, this study was controlled for a number of different confounders including energy intake:BMR, age, sex, education and total food consumed.

Studies showing a non-significant positive association with BMI. One small study of 11- to 13-year-olds in California showed a weak positive association between SSD and BMI that failed to reach significance $(P=0 \cdot 08)^{(27)}$. The review of Malik et al. (5) classifies this study as 'significant positive'. However, this is based on results for diet and regular soda combined, although it is evident that diet soda drives the positive association $(r 0.19 ; P<0.001)$, whereas the association with regular soda was non-significant ( $r 0 \cdot 10 ; P=0 \cdot 08)$ (Table 2).

Studies showing no association with BMI. Thirteen studies showed no association between SSD and BMI, of which twelve were in children (Table 2). The largest was a synthesis of data from thirty-four European countries covering more than 137000 school-aged children ${ }^{(28)}$. The authors reported no association overall between overweight status and soft drinks; OR in adjusted logistic regressions were null (not significantly different from 1) in thirty studies, positive $(>1)$ in two and negative $(<1)$ in two. Most of the studies used a FFQ and assessed overweight from reported BMI using age- and sex-specific cut-offs ${ }^{(29)}$. A further eleven studies in children, including six from the 
$\mathrm{USA}^{(30-34)}$ and five from other countries (Norway ${ }^{(35)}$, $\mathrm{UK}^{(36)}, \operatorname{Spain}^{(37)}$, Brazil $^{(38)}$ and Canada $\left.{ }^{(39)}\right)$, likewise report no association between SSD and overweight. Four of these were new studies not included in previous reviews. Interestingly, both of the studies by Forshee and colleagues ${ }^{(30,31)}$ are classified by Malik et al. as suggestive positive ${ }^{(5)}$. However, in the first study of 3311 young people aged 6-19 years old participating in the Continuing Survey of Food Intake by Individuals (CSFII), there was a positive association with diet soda but not regular soda $(P>0.2)^{(30)}$, while in the second, an analysis of NHANES III using two diet methods, the interpretation of Malik et al. ${ }^{(5)}$ is based on results for the $24 \mathrm{~h}$ recall (slightly positive but $P>0.36$ ) whereas the FFQ data gave results that were slightly negative. In any event both were described by the original author as near zero ${ }^{(31)}$

In agreement with this conclusion, a summary analysis has recently been published of NHANES and CSFII data $(n>34000)$. This found "no substantive differences in BMI and obesity occurrence between frequent and infrequent users of SSD ${ }^{(40)}$. Thus the majority of crosssectional studies do not support a positive association of BMI with SSD.

\section{Longitudinal studies}

Of the seventeen longitudinal studies included, half (eight) showed a significant positive association between SSD consumption and weight or weight gain in at least one subgroup (Table 3). However, four of these showed results which were non-significant in another subgroup ${ }^{(15,41,42)}$, or lost significance on multivariate adjustment for confounders ${ }^{(43)}$.

Longitudinal studies showing a positive association with $B M I$ in children. Five studies were in children (including one in preschool children). In the largest, of more than 12000 children aged 9-14 years old followed up for 2 years ${ }^{(15)}$, consumption of SSD was associated with small (selfreported) BMI gains during the corresponding year, although this was statistically significant only among boys (boys: $+0.03 \mathrm{~kg} / \mathrm{m}^{2}$ per daily serving, $P=0.04$; girls: $+0.02 \mathrm{~kg} / \mathrm{m}^{2}, P=0.096$ ). Boys who increased consumption of sugar-sweetened beverages from the prior year experienced weight gain $\left(+0.04 \mathrm{~kg} / \mathrm{m}^{2}\right.$ per additional daily serving; $P=0.01)$. The authors acknowledge that the magnitude of the actual differences was modest. Thus a boy consuming three servings per $\mathrm{d}$ over 10 years was predicted to gain 0.9 BMI units more than if he consumed none.

In another large American study, among young preschool children from low-income households, consumption of one or more sugar-containing drinks per $\mathrm{d}$ ( $v$. none) was associated with increased risk (OR about 2) of being overweight 1 year later ${ }^{(42)}$. However, this was only statistically significant among children heavier at baseline (BMI $>85$ th percentile). One weakness of this study is that neither parental obesity nor breast-feeding was adjusted for in regressions although both are highly important risk factors for overweight in this age group.

The prospective study by Ludwig et al. ${ }^{(16)}$, although small, has shown the strongest association so far and been particularly influential in driving public policy. Among 548 children (12 years old) in the Planet Health project they reported a significant association between weight gain and SSD consumption (both at baseline and over time), estimating that a single serving was associated with a gain in BMI of $0.24 \mathrm{~kg} / \mathrm{m}^{2}$ over 19 months $(P=0.03)$ and a $60 \%$ increase in odds of obesity ${ }^{(16)}$. The estimates were adjusted for baseline BMI, demographics, diet and lifestyle. The odds of obesity, however, were based on only thirty-seven cases of incident obesity. Although otherwise of good quality, this study has been criticised for using raw BMI rather than BMI $z$-score, which is the preferred measure in children, and for not assessing drinks intake in those who decreased their $\mathrm{BMI}^{(4)}$.

Two further American studies following girls though puberty found that soda consumption significantly predicted increase in BMI. The largest ( $n$ 3371) using the wellrespected National Heart, Lung, and Blood Institute Growth and Health Study employing $3 \mathrm{~d}$ food diaries repeated annually, found that, of all beverages, increasing soda consumption predicted the greatest increase of $\mathrm{BMI}^{(44)}$. However, the investigators did not adjust for physical activity, which has been shown to be a major predictor of weight gain in this cohort ${ }^{(45)}$. The smaller study by Phillips et al. used the FFQ to study 178 girls for 7 years and found that the percentage of energy from soda was associated with higher BMI $z$-score, though not with higher body fat ${ }^{(46)}$.

Longitudinal studies showing a positive association with BMI in adults. The largest study included more than 51000 women nurses ${ }^{(47)}$. Weight gain over a 4 -year period was highest among women who increased their SSD consumption (from one or fewer drinks per week to seven or more per week) and smallest among women who decreased their intake just as dramatically (difference about $3 \mathrm{~kg}$ over 4 years). Interestingly, those with stable consumption patterns (either low or high) did not differ in weight gain (which was also about $3 \mathrm{~kg}$ ).

Similarly, a Mediterranean cohort study in 7194 men and women followed over 28 months found a subgroup effect, with a significant positive association with weight gain only among adults who had been gaining $>3 \mathrm{~kg}$ weight in the 5 years before the study. The adjusted OR for weight gain in the top quintile $v$. lowest quintile of SSD was $1.6(95 \% \mathrm{CI}$ $1 \cdot 2,2 \cdot 1 ; P$ for trend $=0 \cdot 02$ ). This association was absent in the participants who had not gained weight in the 5-year period before baseline ${ }^{(41)}$.

Last, a small Dutch study of 288 middle-aged men studied for 5 years found that one serving of SSD daily was associated with a BMI gain of $0.2 \mathrm{~kg} / \mathrm{m}^{2}$ per year, although this reduced to $0.12 \mathrm{~kg} / \mathrm{m}^{2}$ in the multivariate adjusted model $(P>0.05)^{(43)}$. By comparison the weight gain associated with retiring from an active job was $0.42 \mathrm{~kg} / \mathrm{m}^{2}$.

Longitudinal studies showing a non-significant positive association with BMI in children or adults. Six studies reported a non-significant positive result in at least one group. These include the result among girls in the study by Berkey et al. ${ }^{(15)}(P=0.096)$, the result among normalweight preschoolers in the study by Welsh et al. ${ }^{(42)}$ and that using the final adjusted model in the Dutch study ${ }^{(43)}$. In addition, the study by French et al., which had found a 
small positive association among women at baseline, failed to find this prospectively $(P=0.39$ for women; $P=0.22$ for men) ${ }^{(14)}$ (Table 4). Although Malik et al. ${ }^{(5)}$ classified this study as positive but non-significant, the coefficients were virtually zero (one $12 \mathrm{oz}$ serving (per week) was associated with a weight change of a mere $40 \mathrm{~g}$ for women or $55 \mathrm{~g}$ for men over 2 years).

A recently published study among 244 adolescents (Dortmund Nutritional and Anthropometric Longitudinally Designed (DONALD) cohort), although small, is noteworthy for its strong design (3 d weighed dietary record, measured BMI and 5-year follow-up) and robust statistical analysis (repeated measures, change in BMI on change in intake $)^{(48)}$. Results showed no association with change in BMI z-score, either cross-sectionally or prospectively among boys, but a weak and non-significant association among girls $(P=0 \cdot 08)$, equivalent to an increment of 0.055 BMI SD over 5 years per MJ of SSD (per d). There was a stronger association with fruit juice consumption than with SSD with the result that the combination 'energetic beverages' was statistically significant in girls $(P=0 \cdot 01)$. Finally, one very small study of thirty children followed for 4-8 weeks at summer camp found that those consuming large amounts of SSD $(>16 \mathrm{oz} / \mathrm{d})$ gained more weight $(1.12 \mathrm{~kg})$ than those who consumed less $(0.32-0.48 \mathrm{~kg})^{(49)}$, but the study had insufficient power $(P=0.4)$ and there were other methodological weaknesses, such as recorded weights for only twenty-one children.

Longitudinal studies showing no association with BMI. In the largest study, German adults in the European Prospective Investigation into Cancer and Nutrition (EPIC)-Potsdam cohort ( $n$ 17 369) were followed up over 2 years to assess which food groups were associated with weight gain or weight loss, compared with stable weight ${ }^{(50)}$ (Table 4). 'Soft drinks' included all types including water, SSD, diet drinks and fruit juice. The authors used polytomous logistic regression to examine factors associated with weight gain or weight loss and models were adjusted for a large number of confounders, including physical activity, dieting and baseline BMI. Results were inconclusive for men (higher 'soft drink' intake at baseline was very weakly associated with both losses and gains) and weakly negative for women (slightly greater of risk of large weight losses (OR 1.02; $95 \%$ CI 1.00, 1.03).

Three recent longitudinal studies not included in previous reviews are worth special notice because they assessed fatness, not merely weight or BMI. No association with percentage body fat was found in the DONALD study ${ }^{(48)}$ mentioned above, but the authors suggest that physical activity and timing of pubertal changes may have masked this. The second study, by Mundt et al., tracked body fatness among normal Canadian children and adolescents and found no relationship between fat mass development and consumption of sugar drinks ${ }^{(51)}$. Models included physical activity and adjusted for physical maturation, which is important because soft drink consumption and fat mass both tend to rise with age. Finally, a cohort study of 1203 children in Avon, UK (Avon Longitudinal Study of Parents and Children (ALSPAC) cohort) did not find any significant association between consumption of sugared soft drinks at age 5 or 7 years and fatness at age 9 years $^{(52)}$. This study employed a reliable dietary method and examined body fatness (by dual-energy X-ray absorptiometry) as well as body weight. There was a small positive association with consumption of low energy soft drinks, which were possibly being used as a weight-reduction strategy. A similar positive association with diet drinks but not SSD was found in a study of 166 elementary school children studied for 2 years by Blum et al. ${ }^{(53)}$.

Among 1345 preschool children aged 2-5 years assessed over 6-12 months in Dakota, USA, Newby et al. ${ }^{(54)}$ found no association between change in BMI and consumption of any types of drinks (fruit juice, fruit drinks, milk, soda and diet soda) in multivariate models. Intakes of fruit juice and milk were high in this sample but low intakes and limited variation of soda and fruit drinks in this study may have limited the ability to detect an association with BMI or weight $^{(54)}$.

Finally, in a Norwegian study of 485 older adolescents tracked into adulthood, Kvaavik et al. found no difference in BMI, overweight or obesity at age 33 years between those who were long-term (previous 8 years) high consumers or low consumers of SSD ${ }^{(55)}$. Long-term high SSD consumption was also associated with lifestyle differences including less physical activity, more smoking and higher energy intakes that could cloud associations in this and other studies.

In summary, about half of the longitudinal studies show a significant positive result but the effect appears small. Problems remain in assessing the independent effect of SSD, due to potential confounding from other diet and lifestyle factors.

\section{Intervention studies}

Randomised intervention trials constitute the highest level of evidence for a hypothesis, but these are relatively few (Table 5). Of the four interventions identified, only two were strictly of $\operatorname{SSD}^{(56,57)}$. The first was a pilot study, which randomly assigned 103 adolescents aged $13-18$ years who regularly consumed SSD to either intervention or control groups. The intervention group received home delivery of non-energy beverages for 6 months, during which they decreased consumption of SSD by $82 \%$, while controls did not change. This resulted in a net difference in BMI between groups of $-0.14 \mathrm{~kg} / \mathrm{m}^{2}$ (NS). However, baseline BMI was a significant modifier and among those children who were in the highest third of BMI at baseline the net effect on BMI was $-0.75 \mathrm{~kg} / \mathrm{m}^{2}(P=0.03)$. The authors commented that the greater weight loss with increasing baseline BMI was not simply because of a greater decrease in energy intake from SSD and speculated that some individuals are inherently more susceptible than others to the adverse effects of SSD. The more recent and larger, school-based study of 1140 Brazilian children aged 9-12 years achieved only a small reduction in consumption and produced no difference in weight gain between the intervention and control groups over one school year. However, there was a small effect in those who were already overweight at baseline (about $0 \cdot 2 \mathrm{~kg} / \mathrm{m}^{2}$ ), which was statistically significant among girls $(P=0 \cdot 009)^{(57)}$. 
In England, a randomised cluster intervention by James et al. in six primary schools to reduce consumption of fizzy drinks via education sessions was associated with a $7.7 \%$ difference in the prevalence of obesity between the intervention and control groups at 1 year ${ }^{(9)}$. However, there was no change in mean BMI overall and children in the intervention group still gained weight despite reducing their consumption of fizzy drinks. In a critique of this study, French et al. suggested that methodological limitations leave unanswered questions ${ }^{(58)}$. Only about half of the children returned the beverage diaries and no information was given on other drinks or food or on the reliability or validity of the beverage data. Moreover, this study focused on carbonated drinks exclusively. It is perhaps surprising that such a non-intensive five-session intervention achieved any impact at all, and less surprising that at the 2-year follow-up there was no residual difference between the groups $^{(59)}$.

Finally, the shortest-duration study meeting the present review's criteria was a 10 -week parallel trial investigating the impact of a diet very high in sucrose $(3.4 \mathrm{MJ}$ supplemental energy per d) on ad libitum food intake and weight gain in overweight adults, compared with controls given artificially sweetened foods and drinks. The supplementation was achieved $60 \%$ through sugar-sweetened beverages ${ }^{(60)}$. Energy intake in the sucrose group increased by $1.6 \mathrm{MJ}$ (i.e. about $50 \%$ compensation was observed) and body weight increased in the sucrose group (by $1.6 \mathrm{~kg}$ ) and fell in the sweetener group (by $1.0 \mathrm{~kg}$ ) (difference between groups $P<0.001$ ). Although this clearly shows the potential of excess energy intake from sugar to result in weight gain, actual weight gain was half that predicted from intake. Furthermore, the level of sucrose used in the intervention was extreme $(28 \%$ of energy or approximately twice normal adult intake) and therefore the generalisability of these results is questionable.

\section{Conclusions}

Despite the large number of studies on this topic, the inconsistencies of definition, design, statistical treatment and interpretation make it difficult to draw definitive conclusions as to whether sugar-sweetened beverages are significantly implicated in weight gain. Particular areas of weakness in the evidence base are highlighted below:

(1) Insufficient long-term interventions;

(2) Differing definitions of SSD and terminology;

(3) Differing units for serving size and frequency;

(4) Unreliable methods for dietary assessment;

(5) Narrow focus on SSD with inadequate assessment of other diet components or energy;

(6) Weight and height self-reported, not measured;

(7) Poor or no measurement of physical activity;

(8) Inadequate exploration of confounders or effect modifiers in analysis (for example, baseline BMI, ethnicity, baseline diet, misreporting);

(9) Inconsistent evidence between subgroups;

(10) Underpowered studies, no conclusions can be drawn; (11) Possibility of publication bias towards positive studies.
Cross-sectional studies were the most numerous but have the weakest design in the conventional hierarchy and are prone to confounding and reverse causality. However, they can offer advantages, such as more reliable methods, large sample size and generalisability and, if adjusted for covariates and confounders, may yield results consistent with cohort studies. For example, three longitudinal studies yielded positive associations of similar magnitude when analysed cross-sectionally ${ }^{(14-16)}$ and twelve out of twentyseven cross-sectional studies in the present review found a positive association in at least one group.

Longitudinal studies are more powerful than crosssectional studies, being able to relate change in weight to dietary factors, and often to diet change (change on change). However, they are also prone to confounding from concurrent changes in other aspects of diet and lifestyle and can suffer from attrition bias (drop-outs). Less than half the longitudinal studies reviewed (eight out of seventeen) showed a significant positive effect in at least one subgroup, and four of these had non-significant or null effects in other subgroups. Six studies showed no association in any group. The largest effects tended to be seen in the smaller studies, while larger cohort studies had much more modest effects.

Intervention studies provide the strongest form of evidence and have the potential to infer causality. However, they are also the most costly to conduct. In practice it is often difficult to ensure comparability of groups at baseline, compliance in the intervention group, non-contamination of the control group and adequate monitoring of diet and lifestyle during the trial. Three studies in the present review provide limited evidence that avoidance of sugar-containing soft drinks or substitution with other lower-energy beverages may help prevent further weight gain in overweight individuals ${ }^{(56,57,60)}$. By contrast, little effect was observed in normal-weight individuals. This may be partly explained by lack of power due to small sample size, but also to the small reductions in SSD achieved. Substitution effects (for example, replacement of SSD with fruit juice) may also be a factor in explaining the disappointing outcomes but many studies did not adequately assess consumption of other food and drink.

Previous reviews vary in their conclusions as a result of different inclusion criteria and classification methods. Half regard the evidence as less than persuasive; indeed the total weight of evidence can only be considered in favour of the hypothesis if non-significant studies are counted as positive. At the same time it must be acknowledged that 'absence of evidence is not evidence of absence, ${ }^{(61)}$ and there are methodological reasons that may weaken the ability of studies to demonstrate an effect. In particular, low sample size and measurement error increase the likelihood of type II error (failure to detect an effect where one exists). Dietary methods need to be able to quantify consumption over several days if individual records are to be used.

Variation between studies in how confounding factors are treated also weakens the ability to compare results. Statistical approaches that separate 'energy from soft drinks' and 'energy from other sources' may provide more insights than simple adjustment for total energy in regressions. A surprising number of studies did not 
distinguish between diet and regular versions of soft drinks and thus potentially overestimated positive effects (diet versions being more strongly associated with excess weight). Spurious positive associations could also arise if SSD consumption is correlated with sedentary behaviours, while the reverse could occur if active people consumed more soft drinks. Thus assessment of energy intake and preferably of energy expenditure or physical activity is important in defining the mediators of weight change. It may also help to assess or adjust for confounding resulting from under-reporting.

Finally, there is the issue of publication bias, which may be in either direction. It has been suggested that industryfunded studies tend to show smaller effects ${ }^{(6)}$, but these are comparatively few. On the other hand it is well recognised that publication bias normally works in the opposite direction (null studies remaining unpublished), which results in overestimation of positive effects ${ }^{(7,62)}$.

In conclusion, SSD are by nature a source of energy but there is little evidence from epidemiological studies that they are more obesogenic than any other source of energy. Assertions that SSD are a disproportionate cause of excess body weight and/or that their avoidance would be effective in preventing weight gain are, in my opinion, not well substantiated by the science. The totality of evidence is dominated by American studies that may be less applicable to the European context where consumption is substantially lower and composition or formulation may differ (highfructose corn syrup $v$. sucrose, proportion of diet $v$. non-diet, etc). Most studies suggest that the effect of SSD on body weight is small except in susceptible individuals or at high levels of intake. Thus effects quoted in terms of a $12 \mathrm{oz}$ serving size daily or per MJ of energy exceed those achieved in practice. Moreover, methodological weaknesses mean that many studies cannot detect whether SSD or other aspects of diet and lifestyle have contributed to excess body weight or weight change Meta-analysis provides a way to quantify effect sizes but this requires authors to provide sufficient statistical detail on outcomes and exposures, in subgroups as well as the total sample, and on the effects of adjusting for confounding variables. In particular, more intervention studies are required, especially among overweight consumers of SSD, but these should use reliable measurements of diet and physical activity and have adequate length of follow-up. New trials are due to report soon; therefore ongoing review of this area is imperative. Since obesity is a complex issue whose aetiology involves genetics, diet and lifestyle, it is vital to maintain an integrated perspective on all the influences (and interactions) relevant to weight gain.

\section{Acknowledgements}

S. G. is a public health nutritionist and researcher, commissioned by UNESDA (Union of European Beverages Associations) to conduct an independent review of this topic. The work is entirely that of the author and no representative of the industry had any role in the selection of papers, interpretation of results or commentary on the draft.

\section{References}

1. World Health Organization \& Food and Agriculture Organization (2003) Diet, Nutrition and the Prevention of Chronic Diseases. Geneva: WHO.

2. World Cancer Research Fund (2007) Food, Nutrition, Physical Activity and the Prevention of Cancer. Washington, DC: American Institute for Cancer Research.

3. Bachman CM, Baranowski T \& Nicklas TA (2006) Is there an association between sweetened beverages and adiposity? Nutr Rev 64, 153-174.

4. Pereira MA (2006) The possible role of sugar-sweetened beverages in obesity etiology: a review of the evidence. Int $J$ Obes (Lond) 30, Suppl. 3, S28-S36.

5. Malik VS, Schulze MB \& Hu FB (2006) Intake of sugarsweetened beverages and weight gain: a systematic review. Am J Clin Nutr 84, 274-288.

6. Vartanian LR, Schwartz MB \& Brownell KD (2007) Effects of soft drink consumption on nutrition and health: a systematic review and meta-analysis. Am J Public Health 97, 667-675.

7. Forshee RA, Anderson PA \& Storey ML (2008) Sugarsweetened beverages and body mass index in children and adolescents: a meta-analysis. Am J Clin Nutr 87, 1662-1671.

8. Ruxton C, Gardner E \& McNulty H (2009) Is sugar consumption detrimental to health? A review of the evidence 1995-2006. Crit Rev Food Sci Nutr (In the Press).

9. James J, Thomas P, Cavan D, et al. (2004) Preventing childhood obesity by reducing consumption of carbonated drinks: cluster randomised controlled trial. BMJ 328, 1237-1240.

10. Overby NC, Lillegaard IT, Johansson L, et al. (2004) High intake of added sugar among Norwegian children and adolescents. Public Health Nutr 7, 285-293.

11. Vermunt SHF, Pasman WJ, Schaafsma G, et al. (2003) Effects of sugar intake on body weight: a review. Obes Rev 4, 91-99.

12. Field AE, Austin SB, Gillman MW, et al. (2004) Snack food intake does not predict weight change among children and adolescents. Int J Obes Relat Metab Disord 28, 1210-1216.

13. Flodmark CE, Marcus C \& Britton M (2006) Interventions to prevent obesity in children and adolescents: a systematic literature review. Int J Obes (Lond) 30, 579-589.

14. French SA, Jeffery RW, Forster JL, et al. (1994) Predictors of weight change over two years among a population of working adults: the Healthy Worker Project. Int J Obes Relat Metab Disord 18, 145-154.

15. Berkey CS, Rockett HRH, Field AE, et al. (2004) Sugaradded beverages and adolescent weight change. Obes Res 12, $778-788$.

16. Ludwig DS, Peterson KE \& Gortmaker SL (2001) Relation between consumption of sugar-sweetened drinks and childhood obesity: a prospective, observational analysis. Lancet 357, 505-508.

17. Gibson S \& Neate D (2007) Sugar intake, soft drink consumption and body weight among British children: further analysis of National Diet and Nutrition Survey data with adjustment for under-reporting and physical activity. Int J Food Sci Nutr 58, 445-460.

18. McCarthy SN, Robson PJ, Livingstone MB, et al. (2006) Associations between daily food intake and excess adiposity in Irish adults: towards the development of food-based dietary guidelines for reducing the prevalence of overweight and obesity. Int J Obes (Lond) 30, 993-1002.

19. Troiano RP, Briefel RR \& Carroll MD (2000) Energy and fat intakes of children and adolescents in the United States: data 
from the National Health and Nutrition Examination Surveys. Am J Clin Nutr 72, Suppl. 5, 1343S-1353S.

20. Nicklas TA, Yang SJ, Baranowski T, et al. (2003) Eating patterns and obesity in children. The Bogalusa Heart Study. Am J Prev Med 25, 9-16.

21. Rajeshwari R, Yang SJ, Nicklas TA, et al. (2005) Secular trends in children's sweetened-beverage consumption (1973 to 1994): the Bogalusa Heart Study. J Am Diet Assoc 105, 208-214.

22. Gillis LJ \& Bar-Or O (2003) Food away from home, sugarsweetened drink consumption and juvenile obesity. J Am Coll Nutr 22, 539-545.

23. Novotny R, Daida YG, Acharya S, et al. (2004) Dairy intake is associated with lower body fat and soda intake with greater weight in adolescent girls. J Nutr 134, 1905-1909.

24. Ariza AJ, Chen EH, Binns HJ, et al. (2004) Risk factors for overweight in five- to six-year-old Hispanic-American children: a pilot study. J Urban Health 81, 150-161.

25. Warner ML, Harley K, Bradman A, et al. (2006) Soda consumption and overweight status of 2-year-old MexicanAmerican children in California. Obesity (Silver Spring) 14, 1966-1974.

26. Liebman M, Pelican S, Moore SA, et al. (2003) Dietary intake, eating behavior, and physical activity-related determinants of high body mass index in rural communities in Wyoming, Montana, and Idaho. Int J Obes Relat Metab Disord 27, 684-692.

27. Giammattei J, Blix G, Marshak HH, et al. (2003) Television watching and soft drink consumption: associations with obesity in 11- to 13-year-old schoolchildren. Arch Pediatr Adolesc Med 157, 882-886.

28. Janssen I, Katzmarzyk PT, Boyce WF, et al. (2005) Comparison of overweight and obesity prevalence in school-aged youth from 34 countries and their relationships with physical activity and dietary patterns. Obes Rev 6, 123-132.

29. Cole TJ, Bellizzi MC, Flegal KM, et al. (2000) Establishing a standard definition for child overweight and obesity worldwide: international survey. BMJ 320, 1240-1245.

30. Forshee RA \& Storey ML (2003) Total beverage consumption and beverage choices among children and adolescents. Int J Food Sci Nutr 54, 297-307.

31. Forshee RA, Anderson PA \& Storey ML (2004) The role of beverage consumption, physical activity, sedentary activity and demographics on body mass index of adolescents. Int $J$ Food Sci Nutr 55, 463-478.

32. O'Connor TM, Yang SJ \& Nicklas TA (2006) Beverage intake among preschool children and its effect on weight status. Pediatrics 118, e1010-e1018.

33. Roseman MG, Yeung WK \& Nickelsen J (2007) Examination of weight status and dietary behaviors of middle school students in Kentucky. J Am Diet Assoc 107, 1139-1145.

34. Bandini L, Vu D, Must A, et al. (1999) Comparison of highcalorie, low-nutrient-dense food consumption among obese and non-obese adolescents. Obes Res 7, 438-443.

35. Andersen LF, Lillegaard IT, Overby N, et al. (2005) Overweight and obesity among Norwegian schoolchildren: changes from 1993 to 2000. Scand J Public Health 33, 99-106.

36. Gibson S (1998) Hypothesis: parents may selectively restrict sugar-containing foods for pre-school children with high BMI. Int J Food Sci Nutr 49, 65-70.

37. Rodriguez-Artalejo F, Garcia EL, Gorgojo L, et al. (2003) Consumption of bakery products, sweetened soft drinks and yogurt among children aged 6-7 years: association with nutrient intake and overall diet quality. Br J Nutr 89, $419-429$.
38. Silveira D, Taddei JA, Escrivao MA, et al. (2006) Risk factors for overweight among Brazilian adolescents of low-income families: a case-control study. Public Health Nutr 9, 421-428.

39. Veugelers PJ \& Fitzgerald AL (2005) Prevalence of and risk factors for childhood overweight and obesity. CMAJ 173 , 607-613.

40. Sun SZ \& Empie MW (2007) Lack of findings for the association between obesity risk and usual sugar-sweetened beverage consumption in adults-a primary analysis of databases of CSFII-1989-1991, CSFII-1994-1998, NHANES III, and combined NHANES 1999-2002. Food Chem Toxicol 45, $1523-1536$.

41. Bes-Rastrollo M, Sanchez-Villegas A, Gomez-Gracia E, et al. (2006) Predictors of weight gain in a Mediterranean cohort: the Seguimiento Universidad de Navarra Study 1. Am J Clin Nutr 83, 362-370, quiz 394-395.

42. Welsh JA, Cogswell ME, Rogers S, et al. (2005) Overweight among low-income preschool children associated with the consumption of sweet drinks: Missouri, 1999-2002. Pediatrics 115, e223-e229.

43. Nooyens AC, Visscher TL, Schuit AJ, et al. (2005) Effects of retirement on lifestyle in relation to changes in weight and waist circumference in Dutch men: a prospective study. Public Health Nutr 8, 1266-1274.

44. Striegel-Moore RH, Thompson D, Affenito SG, et al. (2006) Correlates of beverage intake in adolescent girls: the National Heart, Lung, and Blood Institute Growth and Health Study. $J$ Pediatr 148, 183-187.

45. Kimm SY, Glynn NW, Obarzanek E, et al. (2005) Relation between the changes in physical activity and body-mass index during adolescence: a multicentre longitudinal study. Lancet 366, 301-307.

46. Phillips SM, Bandini LG, Naumova EN, et al. (2004) Energydense snack food intake in adolescence: longitudinal relationship to weight and fatness. Obes Res 12, 461-472.

47. Schulze MB, Manson JE, Ludwig DS, et al. (2004) Sugarsweetened beverages, weight gain, and incidence of type 2 diabetes in young and middle-aged women. JAMA 292, 927-934.

48. Libuda L, Alexy U, Sichert-Hellert W, et al. (2008) Pattern of beverage consumption and long-term association with bodyweight status in German adolescents-results from the DONALD study. Br J Nutr 99, 1370-1379.

49. Mrdjenovic G \& Levitsky D (2003) Nutritional and energetic consequences of sweetened drink consumption in 6- to 13year-old children. J Pediatr 142, 604-610.

50. Schulz M, Kroke A, Liese AD, et al. (2002) Food groups as predictors for short-term weight changes in men and women of the EPIC-Potsdam cohort. J Nutr 132, 1335-1340.

51. Mundt CA, Baxter-Jones AD, Whiting SJ, et al. (2006) Relationships of activity and sugar drink intake on fat mass development in youths. Med Sci Sports Exerc 38, $1245-1254$.

52. Johnson L, Mander AP, Jones LR, et al. (2007) Is sugarsweetened beverage consumption associated with increased fatness in children? Nutrition 23, 557-563.

53. Blum JW, Jacobsen DJ \& Donnelly JE (2005) Beverage consumption patterns in elementary school aged children across a two-year period. J Am Coll Nutr 24, 93-98.

54. Newby PK, Peterson KE, Berkey CS, et al. (2004) Beverage consumption is not associated with changes in weight and body mass index among low-income preschool children in North Dakota. J Am Diet Assoc 104, 1086-1094.

55. Kvaavik E, Andersen LF \& Klepp KI (2005) The stability of soft drinks intake from adolescence to adult age and the association between long-term consumption of soft drinks 
and lifestyle factors and body weight. Public Health Nutr $\mathbf{8}$, 149-157.

56. Ebbeling CB, Feldman HA, Osganian SK, et al. (2006) Effects of decreasing sugar-sweetened beverage consumption on body weight in adolescents: a randomized, controlled pilot study. Pediatrics 117, 673-680.

57. Sichieri R, Paula Trotte A, de Souza RA, et al. (2008) School randomised trial on prevention of excessive weight gain by discouraging students from drinking sodas. Public Health Nutr, (epublication ahead of print version 18 June 2008).

58. French SA, Hannan PJ \& Story M (2004) School soft drink intervention study. BMJ 329, E315-E316.
59. James J \& Kerr D (2005) Prevention of childhood obesity by reducing soft drinks. Int J Obes (Lond) 29, Suppl. 2, S54-S57.

60. Raben A, Vasilaras TH, Moller AC, et al. (2002) Sucrose compared with artificial sweeteners: different effects on ad libitum food intake and body weight after $10 \mathrm{wk}$ of supplementation in overweight subjects. Am J Clin Nutr 76, $721-729$.

61. Altman DG \& Bland JM (1995) Absence of evidence is not evidence of absence. BMJ 311, 485.

62. Ioannidis JPA (2005) Why most published research findings are false. PLoS Med 2, e124. 